\title{
Projective representations of spaces of quasianalytic functionals
}

\author{
by \\ José Bonet (Valencia), Reinhold Meise (Düsseldorf) and \\ SERGEĬ N. MELIKHov (Rostov on Don)
}

\begin{abstract}
The weighted inductive limit of Fréchet spaces of entire functions in $N$ variables which is obtained as the Fourier-Laplace transform of the space of analytic functionals on an open convex subset of $\mathbb{R}^{N}$ can be described algebraically as the intersection of a family of weighted Banach spaces of entire functions. The corresponding result for the spaces of quasianalytic functionals is also derived.
\end{abstract}

1. Introduction. Let $\mathcal{A}(G)$ denote the space of all real-analytic functions on an open convex subset $G$ of $\mathbb{R}^{N}$ with complex values. Its strong dual $\mathcal{A}(G)_{b}^{\prime}$ is isomorphic to an (LF)-space $\mathcal{F} \mathcal{A}^{\prime}(G)$ of entire functions on $\mathbb{C}^{N}$ via the Fourier-Laplace transform. Each step space of this (LF)-space is a Fréchet space whose topology is given by weighted sup-norms. Ehrenpreis [8] showed that the topology of $\mathcal{F} \mathcal{A}^{\prime}\left(\mathbb{R}^{N}\right)$ cannot be described by weighted sup-seminorms. Similar questions on the projective description were later investigated by various authors (see Bierstedt [1]). For weighted inductive limits in which the steps are defined by weighted sup-seminorms, one associates a projective hull which is an intersection of weighted Banach spaces. In the projective hull it is possible to perform direct computations and estimates with sup-seminorms. The question whether the projective hull coincides algebraically or topologically with the given inductive limit depends heavily on the properties of the inductive limit.

The first main result of this article is Theorem 3.4, in which we show that the $(\mathrm{LF})$-space $\mathcal{F} \mathcal{A}^{\prime}(G)$ of entire functions coincides with its projective hull algebraically, and has a strictly finer topology. The assertion about the

2000 Mathematics Subject Classification: Primary 46E10; Secondary 46A13, 46F05, $46 \mathrm{~F} 15$.

The research of J. Bonet was partially supported by MCYT and FEDER, Project no. BFM2001-2670 and AVCIT Grupo 03/050. The financial support of the DAAD for a stay of S. N. Melikhov at the University of Düsseldorf in 2001 is acknowledged. S. N. Melikhov is grateful to the Russian Foundation of Basic Research, project no. 02-01-00372, for partial support. 
topology was already proved in Bonet and Meise [4, Theorem 3.1]. The proof of Theorem 3.4 is based on a result about weighted (LB)-spaces of holomorphic functions due to Bierstedt, Meise and Summers [2, Theorem 1.6] and a theorem of Ronkin [18, Theorem 2] about plurisubharmonic functions of at most normal type with respect to order one. The present research also complements our investigations in [5].

The second main result is Theorem 4.6, which shows that Theorem 3.4 can be extended to the spaces $\mathcal{E}_{\{\omega\}}^{\prime}(G)$ of quasianalytic functionals, where $\mathcal{E}_{\{\omega\}}(G)$ denotes the space of all $\omega$-ultradifferentiable functions of Roumieu type on $G$ for a given (quasianalytic) weight function $\omega$ (see 2.1). More precisely, we prove that the $(\mathrm{LF})$-space $\mathcal{F} \mathcal{E}_{\{\omega\}}^{\prime}(G)$ of entire functions on $\mathbb{C}^{n}$ which arise as the Fourier-Laplace transforms of the elements of $\mathcal{E}_{\{\omega\}}(G)_{\mathrm{b}}^{\prime}$ coincides algebraically with its projective hull and has a strictly finer topology. The latter assertion follows again from Bonet and Meise [4, Theorem 3.1]. The corresponding result for non-quasianalytic classes $\mathcal{E}_{\{\omega\}}(G)$ was already obtained by Bonet and Meise [3, Theorem 1]. However, its proof cannot be extended to the present case since the non-quasianalyticity of $\omega$ is applied twice in an essential way. Firstly, it is used to get many functions in $\mathcal{E}_{\{\omega\}}(G)$ with compact support. Secondly, it implies the existence of the harmonic extension of $\omega$ to the upper half-plane which is needed for an application of the global Phragmén-Lindelöf Theorem. In the present (quasianalytic) case we apply a classical estimate of the harmonic measure for the half-disk together with Theorem 3.4 and a recent result of Heinrich and Meise [9] which shows that a theorem of Hörmander [10] on the supports of quasianalytic functionals in $C^{L}\left(\mathbb{R}^{N}\right)^{\prime}$ also holds for elements in $\mathcal{E}_{\{\omega\}}^{\prime}\left(\mathbb{R}^{N}\right)$.

2. Preliminaries. In this preliminary section we introduce the notation that will be used throughout the paper. By $|\cdot|$ we denote the Euclidean norm on $\mathbb{C}^{N}, N \in \mathbb{N}$, while for $a \in \mathbb{C}^{N}$ and $r>0$ we let $B(a, r):=\left\{z \in \mathbb{C}^{N}\right.$ : $|z-a|<r\}$.

2.1. Weight functions. A function $\omega: \mathbb{R} \rightarrow[0, \infty[$ is called a weight function if it is continuous, even, increasing on [0, [, and if it satisfies $\omega(0)=0$ and also the following conditions:

$(\alpha) \omega(2 t)=O(\omega(t))$ as $t$ tends to infinity.

( $\beta) \omega(t)=O(t)$ as $t$ tends to infinity.

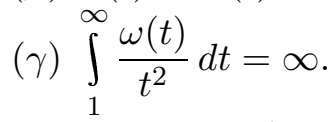

( $\delta) \varphi: t \mapsto \omega\left(e^{t}\right)$ is convex on $[0, \infty[$.

The radial extension $\widetilde{\omega}$ of a weight function $\omega$ is defined as

$$
\widetilde{\omega}: \mathbb{C}^{n} \rightarrow[0, \infty[, \quad \widetilde{\omega}(z):=\omega(|z|) .
$$


It will also be denoted by $\omega$ in what follows, by abuse of notation. The Young conjugate of the function $\varphi=\varphi_{\omega}$, which appears in $(\delta)$, is defined as

$$
\varphi^{*}(x):=\sup \{x y-\varphi(y): y>0\}, \quad x \geq 0 .
$$

2.2. EXAMPLE. The following functions are easily seen to be weight functions:

(1) $\omega(t):=|t|(\log (1+|t|))^{-\alpha}, \quad 0<\alpha \leq 1$.

(2) $\omega(t):=|t|$.

2.3. Spaces of ultradifferentiable functions. Let $\omega$ be a given weight function. For a compact subset $K$ of $\mathbb{R}^{N}$ and $m \in \mathbb{N}$ denote by $C^{\infty}(K)$ the space of all $C^{\infty}$-Whitney jets on $K$ and define

$$
\begin{aligned}
\mathcal{E}_{\{\omega\}}^{m}(K):=\left\{f \in C^{\infty}(K):\right. & \\
& \left.\|f\|_{K, m}:=\sup _{x \in K} \sup _{\alpha \in \mathbb{N}_{0}^{N}}\left|f^{(\alpha)}(x)\right| \exp \left(-\frac{1}{m} \varphi^{*}(m|\alpha|)\right)<\infty\right\} .
\end{aligned}
$$

For an open set $G$ in $\mathbb{R}^{N}$, define the space of all $\omega$-ultradifferentiable functions of Roumieu type on $G$ as

$$
\begin{array}{r}
\mathcal{E}_{\{\omega\}}(G):=\left\{f \in C^{\infty}(G): \text { for each } K \subset G \text { compact there is } m \in \mathbb{N}\right. \\
\text { so that } \left.\|f\|_{K, m}<\infty\right\} .
\end{array}
$$

It is endowed with the topology given by the representation

$$
\mathcal{E}_{\{\omega\}}(G)=\operatorname{proj}_{\leftarrow K} \operatorname{ind}_{m \rightarrow} \mathcal{E}_{\{\omega\}}^{m}(K),
$$

where $K$ runs over all compact subsets of $G$.

Note that $\mathcal{E}_{\{\omega\}}(G)$ is a countable projective limit of (DFN)-spaces which is complete. If $G$ is convex, it follows from Rösner [19, Satz 3.25] and Vogt [21, Theorem 3.4] that $\mathcal{E}_{\{\omega\}}(G)$ is ultrabornological and reflexive. By $\mathcal{E}_{\{\omega\}}(G)^{\prime}$ we denote the strong dual of $\mathcal{E}_{\{\omega\}}(G)$. The elements of $\mathcal{E}_{\{\omega\}}(G)$ are called quasianalytic functions and the elements of $\mathcal{E}_{\{\omega\}}(G)^{\prime}$ are called quasianalytic functionals on $G$.

2.4. REMARK. If $\omega$ is the weight function $\omega(t)=|t|$, then the space $\mathcal{E}_{\{\omega\}}(G)$ coincides with the space $\mathcal{A}(G)$ of all real-analytic functions on $G$. Note that the topology of $\mathcal{A}(G)$ can also be described as the projective limit of the (LB)-spaces $A(K)$, where $K$ runs through all compact subsets of $G$ and $A(K)$ denotes the space of germs of all holomorphic functions on $K$ endowed with its natural inductive limit topology. Martineau [14] proved that $\mathcal{A}(G)$ is ultrabornological.

2.5. Support functions. (a) For a compact set $K$ in $\mathbb{R}^{N}, K \neq \emptyset$, the support function $h_{K}$ of $K$ is defined as

$$
h_{K}: \mathbb{R}^{N} \rightarrow \mathbb{R}, \quad h_{K}(\xi):=\sup _{x \in K}\langle x, \xi\rangle
$$


where $\langle z, w\rangle:=\sum_{j=1}^{N} z_{j} w_{j}$ for $z, w \in \mathbb{C}^{N}$. Obviously, $h_{K}$ is a convex function which is positively homogeneous.

(b) For a compact set $K$ in $\mathbb{C}^{N}$ we define

$$
H_{K}: \mathbb{C}^{N} \rightarrow \mathbb{R}, \quad H_{K}(z):=\sup _{w \in K} \operatorname{Re}\langle z, w\rangle .
$$

2.6. Weighted spaces of holomorphic functions. Let $\omega$ be a weight function and $G$ an open convex subset of $\mathbb{R}^{N}$ which contains the origin. Choose an increasing sequence $\left(K_{n}\right)_{n \in \mathbb{N}}$ of compact subsets of $G$ which satisfy the following conditions: $0 \in \stackrel{\circ}{K}_{1}, K_{n} \subset \stackrel{\circ}{K}_{n+1}$ and $\bigcup_{n \in \mathbb{N}} K_{n}=G$. For $n \in \mathbb{N}$ denote by $h_{n}$ the support function of $K_{n}$ and for $n, k \in \mathbb{N}$ define the weights $v_{n, k} \in C\left(\mathbb{C}^{N}\right)$ by

$$
v_{n, k}(z):=\exp \left(-h_{n}(\operatorname{Im} z)-\frac{1}{k} \omega(z)\right) .
$$

The family $\left(v_{n, k}\right)_{n, k \in \mathbb{N}}$ is denoted by $\mathcal{V}_{\omega, G}$. Next denote by $H\left(\mathbb{C}^{N}\right)$ the space of all holomorphic functions on $\mathbb{C}^{N}$ and, for a given non-negative upper semicontinuous function $v$ on $\mathbb{C}^{N}$, define the weighted seminormed space

$$
H v:=\left\{f \in H\left(\mathbb{C}^{N}\right):\|f\|_{v}:=\sup _{z \in \mathbb{C}^{N}}|f(z)| v(z)<\infty\right\} .
$$

Then the weighted (LF)-space of entire functions associated with $\omega$ and $G$ is defined as

$$
\mathcal{V}_{\omega, G} H:=\operatorname{ind}_{n \rightarrow \operatorname{proj}_{\leftarrow k} H v_{n, k} .}
$$

2.7. Fourier-Laplace transform. Let $\omega$ be a weight function and $G$ a convex open set in $\mathbb{R}^{N}$. It is easy to check that for each $\mu \in \mathcal{E}_{\{\omega\}}(G)^{\prime}$, the Fourier-Laplace transform $\widehat{\mu}$ of $\mu$, defined by

$$
\widehat{\mu}(z):=\mu_{x}(\exp (-i\langle x, z\rangle)),
$$

is in $\mathcal{V}_{\omega, G} H$. In fact, the Fourier-Laplace transform

$$
\mathcal{F}: \mathcal{E}_{\{\omega\}}(G)_{\mathrm{b}}^{\prime} \rightarrow \mathcal{V}_{\omega, G} H, \quad \mathcal{F}(\mu):=\widehat{\mu},
$$

is a linear topological isomorphism. This was shown by Meyer [16] when $N=1$ and in general by Rösner [19, Satz 2.19]. For a simpler proof we refer to Heinrich and Meise [9, Theorem 4.12].

2.8. The projective hull $H \bar{V}_{\omega, G}$. For $\omega, G$, and $\mathcal{V}_{\omega, G}$ as in 2.6 , we define the system $\bar{V}_{\omega, G}$ of weights associated with $\mathcal{V}_{\omega, G}$ according to Bierstedt, Meise and Summers [2] as

$$
\bar{V}_{\omega, G}:=\left\{\bar{v}: \mathbb{C}^{N} \rightarrow[0, \infty[: \bar{v} \text { is upper semicontinuous and }\right.
$$

for each $n \in \mathbb{N}$ there exist $\alpha_{n}>0$ and $k(n) \in \mathbb{N}$ such that $\left.\bar{v} \leq \alpha_{n} v_{n, k(n)}\right\}$. Then the projective hull $H \bar{V}_{\omega, G}$ of $\mathcal{V}_{\omega, G} H$ is defined as

$$
H \bar{V}_{\omega, G}:=\operatorname{proj}_{\leftarrow \bar{v} \in \bar{V}_{\omega, G}} H \bar{v} .
$$


It is easy to check that $H \bar{V}_{\omega, G}$ is a complete locally convex space and that $\mathcal{V}_{\omega, G} H \subset H \bar{V}_{\omega, G}$ with continuous inclusion.

3. Analytic functionals. In this section we will show that the space of Fourier-Laplace transforms of analytic functionals on each convex open set $G$ in $\mathbb{R}^{N}$ coincides algebraically with its projective hull.

3.1. LEMMA. Let $\omega$ be a weight function and $G \subset \mathbb{R}^{N}$ be an open and convex set. Then for each $f \in H \bar{V}_{\omega, G}$ the following holds:

(a) For each $k \in \mathbb{N}$ there exists $A_{k}>0$ such that

$$
|f(x)| \leq A_{k} \exp \left(\frac{1}{k} \omega(x)\right), \quad x \in \mathbb{R}^{N} .
$$

(b) There exist $n \in \mathbb{N}$ and $B>0$ such that

$$
|f(z)| \leq B \exp (n|\operatorname{Im} z|+n \omega(z)), \quad z \in \mathbb{C}^{N} .
$$

Proof. (a) For $k \in \mathbb{N}$ define $\bar{w}_{k}: \mathbb{C}^{N} \rightarrow[0, \infty[$ by

$$
\bar{w}_{k}(z):=0 \quad \text { if } \operatorname{Im} z \neq 0, \quad \bar{w}_{k}(z):=\exp \left(-\frac{1}{k} \omega(z)\right), \quad z \in \mathbb{R}^{N} .
$$

Then for all $n, k \in \mathbb{N}$ we have $\bar{w}_{k} \leq v_{n, k}$. Hence $\bar{w}_{k}$ belongs to $\bar{V}_{\omega, G}$, which implies, for each $f \in H \bar{V}_{\omega, G}$,

$$
\sup _{x \in \mathbb{R}^{N}}|f(x)| \exp \left(-\frac{1}{k} \omega(x)\right)=\sup _{z \in \mathbb{C}^{N}}|f(z)| \bar{w}_{k}(z)=\|f\|_{\bar{w}_{k}}<\infty .
$$

(b) Using the notation introduced in 2.6, choose an increasing sequence $\left(r_{n}\right)_{n \in \mathbb{N}}$ in $\mathbb{N}$ such that $K_{n} \subset \overline{B\left(0, r_{n}\right)}$ for each $n \in \mathbb{N}$. Then define the weight system $\mathcal{W}=\left(w_{n}\right)_{n \in \mathbb{N}}$ by $w_{n}(z):=\exp \left(-r_{n}|\operatorname{Im} z|-n \omega(z)\right)$ and denote the associated weight system according to 2.8 by $\bar{W}$. If $\bar{w} \in \bar{W}$ then for each $n \in \mathbb{N}$ there exists $\alpha_{n}>0$ such that

$$
\begin{aligned}
\bar{w}(z) & \leq \alpha_{n} w_{n}(z)=\alpha_{n} \exp \left(-r_{n}|\operatorname{Im} z|-n \omega(z)\right) \\
& \leq \alpha_{n} \exp \left(-h_{n}(\operatorname{Im} z)-\omega(z)\right)=\alpha_{n} v_{n, 1}(z), \quad z \in \mathbb{C}^{N} .
\end{aligned}
$$

This shows that $\bar{w} \in H \bar{V}_{\omega, G}$ and consequently $H \bar{V}_{\omega, G} \subset H \bar{W}$. Now note that by Bierstedt, Meise and Summers [2, Theorem 1.6], we have $H \bar{W}=$ $\mathcal{W} H$, which implies (b).

3.2. Lemma. Let $\Omega \subset \mathbb{C}^{N}$ be an open convex set and let $G:=\Omega \cap \mathbb{R}^{N}$. Then for each $f \in H \bar{V}_{\omega, G}$ there exist a compact convex set $L \subset \Omega$ and $C>0$ such that

$$
|f(z)| \leq C \exp \left(H_{L}(-i z)\right), \quad z \in \mathbb{C}^{N}
$$

Proof. Choose a fundamental sequence $\left(L_{n}\right)_{n \in \mathbb{N}}$ of compact convex sets for $\Omega$ in such a way that for the given fundamental sequence $\left(K_{n}\right)_{n \in \mathbb{N}}$ of 
compact convex subsets for $G$ used in the definition of the weight system $\mathcal{V}_{\omega, G}$, we have $K_{n} \subset \stackrel{\circ}{L}_{n}$ and $L_{n} \subset \stackrel{\circ}{L}_{n+1}$ for each $n \in \mathbb{N}$. Then choose $k(n) \in \mathbb{N}$ so that

$$
K_{n}+B\left(0, \frac{1}{k(n)}\right) \subset L_{n}, \quad n \in \mathbb{N} .
$$

It is easy to check that this choice implies

$$
H_{K_{n}}(z)+\frac{|z|}{k(n)} \leq H_{L_{n}}(z), \quad z \in \mathbb{C}^{N}, n \in \mathbb{N} .
$$

Next note that

$$
\begin{aligned}
h_{n}(\operatorname{Im} z) & =h_{K_{n}}(\operatorname{Im} z)=\sup _{y \in K_{n}}\langle\operatorname{Im} z, y\rangle=\sup _{y \in K_{n}} \operatorname{Re}\langle\operatorname{Im} z-i \operatorname{Re} z, y\rangle \\
& =\sup _{y \in K_{n}} \operatorname{Re}\langle-i(\operatorname{Re} z+i \operatorname{Im} z), y\rangle=H_{K_{n}}(-i z) .
\end{aligned}
$$

Obviously, these estimates imply

$$
-H_{L_{n}}(-i z) \leq-h_{n}(\operatorname{Im} z)-\frac{|z|}{k(n)}, \quad z \in \mathbb{C}^{N}, n \in \mathbb{N} .
$$

Now define the weight system $\mathcal{U}:=\left(u_{n}\right)_{n \in \mathbb{N}}$, where

$$
u_{n}(z):=\exp \left(-H_{L_{n}}(-i z)\right), \quad z \in \mathbb{C}^{N},
$$

and denote by $\bar{U}$ the associated weight system, according to 2.8. If we fix $\bar{u} \in \bar{U}$ then for each $n \in \mathbb{N}$ there exists $\alpha_{n}>0$ such that $\bar{u} \leq \alpha_{n} u_{n}$. Since $\omega$ satisfies condition $2.1(\beta)$, we can choose $D \geq 1$ such that $\omega(t) \leq D|t|+D$ for all $t \in \mathbb{R}$. Then it follows from (3.1) that for each $n \in \mathbb{N}$ we have

$$
\begin{aligned}
\bar{u}(z) & \leq \alpha_{n} u_{n}(z) \leq \alpha_{n} \exp \left(-h_{n}(\operatorname{Im} z)-\frac{|z|}{k(n)}\right) \\
& \leq \alpha_{n} \exp \left(-h_{n}(\operatorname{Im} z)-\frac{\omega(z)}{D k(n)}+\frac{1}{k(n)}\right) \leq e \alpha_{n} v_{n, D k(n)}(z) .
\end{aligned}
$$

This implies $\bar{u} \in \bar{V}_{\omega, G}$. Hence $|f| \bar{u}$ is bounded on $\mathbb{C}^{N}$ for each $f \in H \bar{V}_{\omega, G}$. Now note that for each $n \in \mathbb{N}$ there exists $\varepsilon_{n}>0$ such that $L_{n}+\overline{B\left(0, \varepsilon_{n}\right)} \subset$ $L_{n+1}$. This implies $H_{L_{n+1}}(z) \geq H_{L_{n}}(z)+\varepsilon_{n}|z|$ and consequently $-H_{L_{n+1}}(z)$ $+H_{L_{n}}(z) \leq-\varepsilon_{n}|z|, z \in \mathbb{C}^{N}$. By the definition of $\mathcal{U}$, this shows that we can apply Bierstedt, Meise, and Summers [2, Theorem 1.3(d)] to get $\mathcal{U} H=H \bar{U}$. This implies that for each $f \in H \bar{V}_{\omega, G} \subset H \bar{U}$ there is $n \in \mathbb{N}$ such that $|f| u_{n}$ is bounded on $\mathbb{C}^{N}$. By the definition of $u_{n}$, this completes the proof of the lemma.

To apply Lemmas 3.1 and 3.2 we need a result about plurisubharmonic functions $u$ defined on $\mathbb{C}^{N}$. Recall that $u$ is said to be of at most normal type with respect to order 1 if there are constants $A, B>0$ such that

$$
u(z) \leq A|z|+B, \quad z \in \mathbb{C}^{N} .
$$


The following theorem was proved by Ronkin [18, Theorem 2] (a similar result was obtained by Napalkov [17, Theorem 15.21]).

3.3. THEOREM. Let u be a plurisubharmonic function of at most normal type with respect to order 1 and let $\alpha$ be a continuous function on $\mathbb{R}^{N}$ which is positively homogeneous with real values. If for each $\varepsilon>0$ there exist $C_{\varepsilon}^{(1)}, C_{\varepsilon}^{(2)}>0$ such that

$$
u(x) \leq \varepsilon|x|+C_{\varepsilon}^{(1)}, \quad x \in \mathbb{R}^{N}, \quad u(i y) \leq \alpha(y)+\varepsilon|y|+C_{\varepsilon}^{(2)}, \quad y \in \mathbb{R}^{N},
$$

then for each $\varepsilon>0$ there exists $C_{\varepsilon}^{(3)}>0$ such that

$$
u(x+i y) \leq \alpha(y)+\varepsilon(|x|+|y|)+C_{\varepsilon}^{(3)}, \quad x, y \in \mathbb{R}^{N} .
$$

3.4. TheOREM. For each open convex set $G \subset \mathbb{R}^{N}$ and $\omega(t)=|t|$ for $t \in \mathbb{R}$, we have $\mathcal{V}_{\omega, G} H=H \bar{V}_{\omega, G}$, while the topology of $\mathcal{V}_{\omega, G} H$ is strictly finer than the one of $H \bar{V}_{\omega, G}$.

Proof. Since $\mathcal{V}_{\omega, G} H \subset H \bar{V}_{\omega, G}$ we only have to prove the converse inclusion. To do so it is no restriction to assume $0 \in G$. Then fix $f \in H \bar{V}_{\omega, G}$ and note that by Lemma 3.1(b), $f$ is of exponential type. Next let $\Omega:=G+i \mathbb{R}^{N}$. By Lemma 3.2 there exists a compact set $L$ in $\Omega$ and $C>0$ such that

$$
|f(z)| \leq C \exp \left(H_{L}(-i z)\right), \quad z \in \mathbb{C}^{N} .
$$

Obviously, we can choose $m \in \mathbb{N}$ so that $L \subset K_{m}+i \mathbb{R}^{N}$. This implies $H_{L}(y) \leq h_{m}(y)$ for each $y \in \mathbb{R}^{N}$ and consequently

$$
|f(i y)| \leq C \exp \left(H_{L}(y)\right) \leq C \exp \left(h_{m}(y)\right), \quad y \in \mathbb{R}^{N} .
$$

Now note that by $3.1(\mathrm{a})$, for each $\varepsilon>0$ there exists $B>0$ such that

$$
|f(x)| \leq B \exp (\varepsilon|x|), \quad x \in \mathbb{R}^{N} .
$$

Hence the function $u: \mathbb{C}^{N} \rightarrow[-\infty, \infty[, u(z):=\log |f(z)|$, satisfies the hypotheses of Theorem 3.3. Therefore, this theorem implies that for each $\varepsilon>0$ there is $B_{\varepsilon}>0$ such that

$$
|f(z)| \leq B_{\varepsilon} \exp \left(h_{m}(\operatorname{Im} z)+\varepsilon|z|\right), \quad z \in \mathbb{C}^{N} .
$$

Obviously, this estimate implies $f \in \mathcal{V}_{\omega, G} H$ and hence $H \bar{V}_{\omega, G} \subset \mathcal{V}_{\omega, G} H$.

The second assertion of the theorem was proved in [4, Theorem 3.1].

4. Quasianalytic functionals. In this section we show that Theorem 3.4 extends to arbitrary weight functions $\omega$. To achieve this we use a lemma which follows from estimates of the harmonic measure of the upper half-disk, as stated in Braun, Meise, and Vogt [7, Lemma 4.1].

4.1. Lemma. Let $\mu, a, b, R>0$, let $z \in \mathbb{C}$ with $|\operatorname{Im} z| \leq R$, and let $\varphi$ be subharmonic in a neighborhood of $D=\{\zeta \in \mathbb{C}:|\zeta-\operatorname{Re} z| \leq R\}$ satisfying

(1) $\varphi(\zeta) \leq \mu|\operatorname{Im} \zeta|+a R$ for all $\zeta \in D$.

(2) $\varphi(\xi) \leq b R$ for all $\xi \in D \cap \mathbb{R}$. 
Then $\varphi$ satisfies

(3) $\varphi(z) \leq \frac{4}{\pi}(\mu+a)|\operatorname{Im} z|+b R$.

4.2. LEMMA. Let $\omega$ be a weight function and $G$ an open convex set in $\mathbb{R}^{N}$ with $0 \in G$. Then for each $f \in H \bar{V}_{\omega, G}$ there exists $m \in \mathbb{N}$ such that for each $k \in \mathbb{N}$ there exists $A_{k}>0$ such that

$$
|f(z)| \leq A_{k} \exp \left(m|\operatorname{Im} z|+\frac{1}{k} \omega(z)\right), \quad z \in \mathbb{C}^{N} .
$$

Proof. Fix $f \in H \bar{V}_{\omega, G}$. By Lemma 3.1(a),

$$
\log |f(x)|=o(\omega(x)), \quad x \in \mathbb{R}^{N}, \quad \text { as }|x| \rightarrow \infty .
$$

We apply Braun, Meise, and Taylor [6, 1.7 and 1.8(a)] to find a weight $\sigma$ such that $\sigma=o(\omega)$ and $A^{\prime}>0$ with

$$
|f(x)| \leq A^{\prime} \exp (\sigma(x)), \quad x \in \mathbb{R}^{N} .
$$

Now, by Lemma 3.1(b), there are $n \in \mathbb{N}$ and $B>0$ with

$$
|f(z)| \leq B \exp (n|\operatorname{Im} z|+n \omega(z)), \quad z \in \mathbb{C}^{N} .
$$

Fix $A>\max \left(B, A^{\prime}, 1\right)$. The function $\varphi$ defined by $\varphi(z):=\log |f(z)|-\log A$ is plurisubharmonic on $\mathbb{C}^{N}$ and satisfies the inequalities

$$
\begin{aligned}
& \varphi(z) \leq n|\operatorname{Im} z|+n \omega(z), \quad z \in \mathbb{C}^{N}, \\
& \varphi(x) \leq \sigma(x), \quad x \in \mathbb{R}^{N} .
\end{aligned}
$$

Select $C, t_{0}>0$ such that for each $t \geq t_{0}$ we have

$$
\begin{array}{ll}
\omega(2 t) \leq C \omega(t), \quad \sigma(2 t) \leq C \sigma(t), \quad \omega(t) \leq C t, \quad \sigma(t) \leq C t, \\
\omega(t) \leq C|t|+C \quad \text { for all } t \in \mathbb{R} .
\end{array}
$$

We fix $\zeta \in \mathbb{C}^{N}$ and distinguish three cases.

CASE (A). Suppose that $|\operatorname{Im} \zeta| \geq|\operatorname{Re} \zeta|$. Then $|\zeta| \leq 2|\operatorname{Im} \zeta|$ and, by (4.1) and by (4.3),

$$
\begin{aligned}
\varphi(\zeta) & \leq n|\operatorname{Im} \zeta|+n \omega(\zeta) \leq n|\operatorname{Im} \zeta|+n C|\zeta|+n C \\
& \leq n|\operatorname{Im} \zeta|+2 n C|\operatorname{Im} \zeta|+n C .
\end{aligned}
$$

CASE (B). Suppose that $|\operatorname{Im} \zeta|<|\operatorname{Re} \zeta| \leq t_{0}$. Now $|\zeta| \leq|\operatorname{Im} \zeta|+|\operatorname{Re} \zeta| \leq$ $2|\operatorname{Re} \zeta| \leq 2 t_{0}$ and

$$
\varphi(\zeta) \leq n|\operatorname{Im} \zeta|+n \omega(\zeta) \leq n|\operatorname{Im} \zeta|+n \omega\left(2 t_{0}\right)
$$

CASE (C). Suppose that $|\operatorname{Im} \zeta|<|\operatorname{Re} \zeta|$ and $|\operatorname{Re} \zeta|>t_{0}$. We set $x:=$ $\operatorname{Re} \zeta, y:=\operatorname{Im} \zeta$ and $\Omega:=\left\{\vartheta \in \mathbb{C}^{N}:|\vartheta-x| \leq|x|\right\}$. Since $|\zeta-x|=|y|<|x|$ we have $\zeta \in \Omega$. If $\vartheta \in \Omega$ then

$$
|\vartheta| \leq|\vartheta-x|+|x| \leq 2|x|
$$


and, by (4.3),

$$
\omega(\vartheta)=\omega(|\vartheta|) \leq \omega(2|x|) \leq C \omega(x) .
$$

We apply (4.1) to conclude that

$$
\varphi(\vartheta) \leq n|\operatorname{Im} \vartheta|+n \omega(\vartheta) \leq n|\operatorname{Im} \vartheta|+n C \omega(x) .
$$

On the other hand, if $\xi \in \mathbb{R}^{N} \cap \Omega$, we deduce from (4.2) that

$$
\varphi(\xi) \leq \sigma(\xi)=\sigma(|\xi|) \leq \sigma(2|x|) \leq C \sigma(x) .
$$

For $\zeta \in \mathbb{C}$ with $\operatorname{Im} \zeta \neq 0$ define the plurisubharmonic function

$$
\psi(u):=\varphi\left(x+u \frac{\operatorname{Im} \zeta}{|\operatorname{Im} \zeta|}\right), \quad u \in \mathbb{C} .
$$

We will apply Lemma 4.1 to the function $\psi$ on $D:=\{u \in \mathbb{C}:|u| \leq|x|\}$. If $u \in D$ we have $x+u \operatorname{Im} \zeta /|\operatorname{Im} \zeta| \in \Omega$. From $|\operatorname{Im} \zeta|<|\operatorname{Re} \zeta|=|x|$ it follows that $u_{0}:=i|\operatorname{Im} \zeta| \in D$. In addition $x+u_{0} \operatorname{Im} \zeta /|\operatorname{Im} \zeta|=x+i \operatorname{Im} \zeta=\zeta$. From (4.4) we get

$$
\begin{aligned}
\psi(u) & =\varphi\left(x+u \frac{\operatorname{Im} \zeta}{|\operatorname{Im} \zeta|}\right) \leq n\left|\operatorname{Im}\left(x+u \frac{\operatorname{Im} \zeta}{|\operatorname{Im} \zeta|}\right)\right|+n C \omega(x) \\
& =n|\operatorname{Im} u|+n C \omega(x), \quad u \in D
\end{aligned}
$$

From (4.5) we conclude that

$$
\psi(u)=\varphi\left(x+u \frac{\operatorname{Im} \zeta}{|\operatorname{Im} \zeta|}\right) \leq C \sigma(x), \quad u \in D \cap \mathbb{R} .
$$

By Lemma 4.1 with $\mu:=n, R:=|x|, a:=n C \omega(x) /|x|, b:=C \sigma(x) /|x|, z:=$ $u_{0}$, and by (4.3), we have

$\psi\left(u_{0}\right) \leq \frac{4}{\pi}\left(n+n C \frac{\omega(x)}{|x|}\right)\left|\operatorname{Im} u_{0}\right|+C \sigma(x) \leq \frac{4}{\pi}\left(n+n C^{2}\right)|\operatorname{Im} \zeta|+C \sigma(\operatorname{Re} \zeta)$.

Hence

$$
\varphi(\zeta) \leq \frac{4}{\pi}\left(n+n C^{2}\right)|\operatorname{Im} \zeta|+C \sigma(\zeta)
$$

Summarizing Cases (A), (B) and (C), and setting $m \geq \max (2 n C+n$, $\left.\frac{4}{\pi}\left(n+n C^{2}\right)\right), T \geq \max \left(n C, n \omega\left(2 t_{0}\right)\right)$ we conclude

$$
|\varphi(\zeta)| \leq m|\operatorname{Im} \zeta|+C \sigma(\zeta)+T, \quad \zeta \in \mathbb{C}^{N}
$$

therefore

$$
|f(\zeta)| \leq A e^{T} \exp (m|\operatorname{Im} \zeta|+C \sigma(\zeta)), \quad \zeta \in \mathbb{C}^{N}
$$

Since $\sigma=o(\omega)$, this implies that for each $k \in \mathbb{N}$ there exists $A_{k}>0$ such that

$$
|f(z)| \leq A_{k} \exp \left(m|\operatorname{Im} z|+\frac{1}{k} \omega(z)\right), \quad z \in \mathbb{C}^{N}
$$


4.3. Analytic functionals with real carrier. For a compact set $K$ in $\mathbb{C}^{N}$ the space $A^{\prime}(K)$ is defined as the set of all $u \in H\left(\mathbb{C}^{N}\right)^{\prime}$ which have the property that for each open neighborhood $G$ of $K$ in $\mathbb{C}^{N}$ there exists $C_{G}>0$ such that

$$
|u(f)| \leq C_{G} \sup _{z \in G}|f(z)|, \quad f \in H\left(\mathbb{C}^{N}\right) .
$$

The elements of $A^{\prime}(K)$ are called analytic functionals carried by $K$. It is well known that in general there is no minimal carrier for a given $u \in A^{\prime}(K)$. However, if we let

$$
A^{\prime}\left(\mathbb{R}^{N}\right):=\bigcup\left\{A^{\prime}(K): K \subset \mathbb{R}^{N} \text { compact }\right\}
$$

then for each $u \in A^{\prime}\left(\mathbb{R}^{N}\right)$ there is a smallest compact set $K \subset \mathbb{R}^{N}$ such that $u \in A^{\prime}(K)$ (see Schapira [20, Theorem 111] or Hörmander [11, Theorem 9.1.6]). This set $K$ is called the support of $u$. From Hörmander [12, Theorem 15.1.5], it follows that for a compact convex set $K$ in $\mathbb{R}^{N}$, an entire function $f \in H\left(\mathbb{C}^{N}\right)$ is the Fourier-Laplace transform of some $u \in A^{\prime}(K)$ if and only if $f$ satisfies the following estimate: For each $\varepsilon>0$ there exists $C_{\varepsilon}>0$ such that

$$
|f(z)| \leq C_{\varepsilon} \exp \left(h_{K}(\operatorname{Im} z)+\varepsilon|z|\right), \quad z \in \mathbb{C}^{N} .
$$

4.4. LEMMA. For each weight function $\omega$ and each open convex set $G \subset$ $\mathbb{R}^{N}$ the restriction map $\varrho: H\left(\mathbb{C}^{N}\right) \rightarrow \mathcal{E}_{\{\omega\}}(G)$ is linear, continuous, and has dense range. Hence $\varrho^{t}: \mathcal{E}_{\{\omega\}}(G)_{\mathrm{b}}^{\prime} \rightarrow H\left(\mathbb{C}^{N}\right)_{\mathrm{b}}^{\prime}$ is injective and im $\varrho^{t} \subset$ $A^{\prime}\left(\mathbb{R}^{N}\right)$.

Proof. Since $\omega$ satisfies condition $2.1(\beta)$, one can check that $\mathcal{A}(G)$ is continuously embedded into $\mathcal{E}_{\{\omega\}}(G)$. Since $\varrho$ factors through $\mathcal{A}(G)$, the continuity of $\varrho$ follows. By the result of Rösner stated in 2.7, the set $\left\{f_{z}: z \in \mathbb{C}^{N}\right\}$, where $f_{z}$ is defined by $f_{z}(x):=\exp (-i\langle x, z\rangle)$, is dense in $\mathcal{E}_{\{\omega\}}(G)$. Hence $\varrho\left(H\left(\mathbb{C}^{N}\right)\right)$ is dense in $\mathcal{E}_{\{\omega\}}(G)$ and consequently $\varrho^{t}$ is injective. Obviously, the continuity estimate for $u$ implies that $\varrho^{t}(u)$ is in $A^{\prime}\left(\mathbb{R}^{N}\right)$.

To prove the main theorem of this section we will use the following result of Heinrich and Meise [9] which shows that Theorem 3.4 of Hörmander [10] on the supports of quasianalytic functionals also holds for the spaces $\mathcal{E}_{\{\omega\}}(G)$ considered here.

4.5. TheOREM. Let $\omega$ be a weight function, let $u \in \mathcal{E}_{\{\omega\}}^{\prime}\left(\mathbb{R}^{N}\right)$, and let $\Omega$ be an open subset of $\mathbb{R}^{N}$. If the support of $\varrho^{t}(u)$ is contained in $\Omega$ then there exists a unique element $U \in \mathcal{E}_{\{\omega\}}^{\prime}(\Omega)$ which satisfies $\left.U\right|_{\mathcal{E}_{\{\omega\}}\left(\mathbb{R}^{N}\right)}=u$.

4.6. TheOREM. For each weight function $\omega$ and each convex open set $G$ in $\mathbb{R}^{N}$ the spaces $\mathcal{V}_{\omega, G} H$ and $H \bar{V}_{\omega, G}$ coincide as vector spaces, while the topology of $\mathcal{V}_{\omega, G} H$ is strictly finer than the one of $H \bar{V}_{\omega, G}$. 
Proof. Since $\mathcal{V}_{\omega, G} H \subset H \bar{V}_{\omega, G}$ we only have to prove the converse inclusion. It is no restriction to assume $0 \in G$. If we fix $f \in H \bar{V}_{\omega, G}$ then it follows from Lemma 4.2 that $f \in \mathcal{V}_{\omega, \mathbb{R}^{N}} H$. By 2.7 there exists $u \in \mathcal{E}_{\{\omega\}}^{\prime}\left(\mathbb{R}^{N}\right)$ such that $f=\mathcal{F}(u)$. Next define $\sigma(t):=|t|, t \in \mathbb{R}$, and note that $\omega=O(\sigma)$ by hypothesis. From this it follows easily that $\bar{V}_{\sigma, G} \subset \bar{V}_{\omega, G}$. Hence $f$ belongs to $H \bar{V}_{\sigma, G}=\mathcal{V}_{\sigma, G} H$, by Theorem 3.4. Therefore, it follows from (4.6) that $f=\mathcal{F}(v)$, where $v \in \mathcal{E}_{\{\sigma\}}(G)^{\prime}=\mathcal{A}(G)^{\prime}$. Consequently, there exists $K \subset G$ compact with $K$ equal to the support of $v$. By Lemma 4.4 we have $v=\varrho^{t}(u)$. By the result of Heinrich and Meise [9] stated in Theorem 4.5, this implies $u \in \mathcal{E}_{\{\omega\}}(G)^{\prime}$ and hence $f \in \mathcal{V}_{\omega, G} H$.

The second statement of the theorem was proved in Bonet and Meise [4, Theorem 3.1].

We conclude this section with further examples of weight functions which extend Example 2.2(1).

4.7. ExAmple. For $k \in \mathbb{N}_{0}$ define recursively

$$
e_{0}:=1, \quad e_{k}:=\exp \left(e_{k-1}\right), \quad \log _{0} x:=x, \quad \log _{k} x:=\log \left(\log _{k-1} x\right) .
$$

For $s \in \mathbb{N}$ let $\alpha \in] 0,1]$ if $s=1$ and $\alpha>0$ if $s \geq 2$ and define

$$
\omega_{\alpha, s}: \mathbb{R} \rightarrow\left[0, \infty\left[, \quad \omega_{\alpha, s}(t):=\frac{|t|}{\left(\log _{s}\left(e_{s}+|t|\right)\right)^{\alpha}} .\right.\right.
$$

Then it is easy to check that $\omega_{\alpha, s}$ is a weight function for each $s \in \mathbb{N}$ and $\alpha$ in the range defined above. Hence we have $\mathcal{V}_{\omega_{\alpha, s}, G} H=H \bar{V}_{\omega_{\alpha, s}, G}$ for each convex open set $G$ in $\mathbb{R}^{N}$.

\section{References}

[1] K. D. Bierstedt, A survey of some results and open problems in weighted inductive limits and projective description for spaces of holomorphic functions, Bull. Soc. Roy. Sci. Liège 70 (2001), 167-182.

[2] K. D. Bierstedt, R. Meise and W. H. Summers, A projective description of weighted inductive limits, Trans. Amer. Math. Soc. 272 (1982), 107-160.

[3] J. Bonet and R. Meise, Ultradistributions of Roumieu type and projective descriptions, J. Math. Anal. Appl. 255 (2001), 122-136.

[4] -, -, Quasianalytic functionals and projective descriptions, Math. Scand., to appear.

[5] J. Bonet, R. Meise and S. N. Melikhov, Holomorphic functions on locally closed convex sets and projective descriptions, Bull. Belg. Math. Soc. Simon Stevin 10 (2003), 491-503.

[6] R. W. Braun, R. Meise and B. A. Taylor, Ultradifferentiable functions and Fourier analysis, Results Math. 17 (1990), 206-237.

[7] R. W. Braun, R. Meise and D. Vogt, Characterization of the linear partial differential operators with constant coefficients which are surjective on non-quasianalytic classes of Roumieu type on $\mathbb{R}^{N}$, Math. Nachr. 168 (1994), 19-54. 
[8] L. Ehrenpreis, Solution of some problem of division. Part IV. Invertible and elliptic operators, Amer. J. Math. 82 (1960), 522-588.

[9] T. Heinrich and R. Meise, A theorem of supports for quasianalytic functionals, preprint.

[10] L. Hörmander, Between distributions and hyperfunctions, Astérisque 131 (1985), 89-106.

[11] - The Analysis of Linear Partial Differential Operators I, Springer, Berlin, 1983.

[12] —, The Analysis of Linear Partial Differential Operators II, Springer, Berlin, 1983.

[13] H. Komatsu, Ultradistributions I. Structure theorems and a characterization, J. Fac. Sci. Univ. Tokyo Sect. IA Math. 20 (1973), 25-105.

[14] A. Martineau, Sur la topologie des espaces de fonctions holomorphes, Math. Ann. 163 (1966), 62-88.

[15] R. Meise and D. Vogt, Introduction to Functional Analysis, Oxford Univ. Press, New York, 1997.

[16] T. Meyer, Die Fourier-Laplace Transformation quasianalytischer Funktionale und ihre Anwendung auf Faltungsoperatoren, Diplomarbeit, Düsseldorf, 1989.

[17] V. V. Napalkov, Convolution Equations in Multidimensional Spaces, Nauka, Moscow, 1982 (in Russian).

[18] L. I. Ronkin, Remarks on theorems of Phragmén-Lindelöf type for analytic functions of several variables, Teor. Funktsiǔ Funktsional. Anal. i Prilozhen. 15 (1972), 97-102 (in Russian).

[19] T. Rösner, Surjektivität partieller Differentialoperatoren auf quasianalytischen Roumieu-Klassen, Dissertation, Düsseldorf, 1997.

[20] P. Schapira, Théorie des hyperfonctions, Lecture Notes in Math. 126, Springer, Berlin, 1970.

[21] D. Vogt, Topics on projective spectra of (LB)-spaces, in: Advances in the Theory of Fréchet Spaces, T. Terzioğlu (ed.), Kluwer, Dordrecht, 1988, 11-27.

Departamento de Matemática Aplicada

Universidad Politécnica de Valencia

E-46071 Valencia, Spain

E-mail: jbonet@mat.upv.es

Department of Mechanics and Mathematics

Rostov State University

Zorge St. 5

344090 Rostov on Don, Russia

E-mail: melih@ms.math.rsu.ru
Mathematisches Institut Heinrich-Heine-Universität Universitätsstraße 1

D-40225 Düsseldorf, Germany E-mail: meise@math.uni-duesseldorf.de

Received October 7, 2003

Revised version April 6, 2004 